\title{
Resolution Behavior of cis- and trans-Octadecenoic Acid Isomers by AOCS Official Method Using SP-2560 Column
}

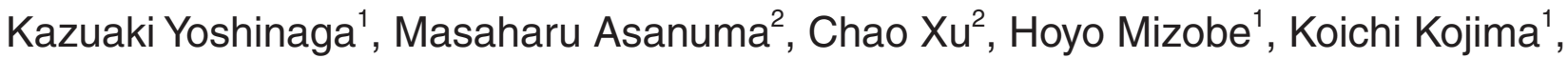 \\ Toshiharu Nagai ${ }^{1}$, Fumiaki Beppu ${ }^{2}$ and Naohiro Gotoh ${ }^{2 *}$ \\ ${ }^{1}$ Tsukishima Foods Industry Co. Ltd. (3-17-9, Higashi Kasai, Edogawa-ku, Tokyo 134-8520, Japan) \\ ${ }^{2}$ Department of Food Science and Technology, Tokyo University of Marine Science and Technology (4-5-7 Konan, Minato-ku, Tokyo 108-8477, \\ Japan)
}

\begin{abstract}
The gas chromatography-flame ionization detector equipped with a higher polarity column (i.e., SP-2560) has often been used for the quantification of trans-fatty acids in food. In particular, AOCS Ce 1h05, the official method of the American Oil Chemists' Society (AOCS), is a highly effective method to separate the isomers of trans-fatty acids. In this study, the resolution behavior and the response factors of cis- and trans-octadecenoic acid methyl ester (C18:1-ME) isomers separated by the AOCS Ce 1h-05 method were investigated, and the contents of each cis- and trans-C18:1-ME isomer in partially hydrogenated vegetable oil (PHVO) and milk fat were quantified by using the calibration curves obtained for the respective isomers. The relative response factors for the trans- and cis-C18:1-ME isomers against the internal standard heneicosanoic acid methyl ester (C21:0-ME) were $1.031 \pm 0.040$ (mean \pm SD) and $0.990 \pm$ 0.032, respectively. The relative response factors of trans-isomers tend to be higher than those of cis-C18:1ME isomers. The peaks of cis-4-C18:1-ME, cis-5-C18:1-ME, cis-6-C18:1-ME, cis-7-C18:1-ME, cis-8-C18:1ME, and cis-9-C18:1-ME isomers overlapped with those of trans-C18:1-ME isomers. Both PHVO and milk fat contained many types of cis- and trans-C18:1 isomers, and the total contents of the trans-C18:1 isomer in PHVO and milk fat were $28.01 \mathrm{~g}$ and $3.62 \mathrm{~g}$ per $100 \mathrm{~g}$ oil, respectively. When the trans-C18:1-ME isomer was separated from the cis-C18:1-ME by using a silver-ion cartridge column before the analyses, the total contents of the trans-C18:1 isomer in PHVO and milk fat were $23.03 \mathrm{~g}$ and $2.78 \mathrm{~g}$ per $100 \mathrm{~g}$ oil, respectively. The difference in the trans-C18:1 isomer content between the two methods was ascribed to the partial overlapping of cis-isomer peaks with the peaks of trans-C18:1-ME isomers, in the chromatogram.
\end{abstract}

Key words: AOCS official method, GC-FID, Resolution, Trans-fatty acid isomer

\section{INTRODUCTION}

Trans-fatty acids are the geometrical isomers of monounsaturated and polyunsaturated fatty acids having nonconjugated, interrupted by at least one methylene group, carbon-carbon double bonds in the trans configuration ${ }^{1)}$. The primary trans-fatty acid isomers in foods consist of 13 octadecenoic acid (C18:1) isomers with double-bond positions from $\Delta 4$ to $\Delta 16^{2)}$. The two major ways in which dietary trans-fatty acids are formed are partial hydrogenation of vegetable oil and bacterial isomerization in the rumen of ruminants ${ }^{3)}$. In particular, partially hydrogenated vegetable oil (PHVO) characteristically contains a high percentage of elaidic acid (trans-9-C18:1 or trans-C18:1n-9) ${ }^{2}$.
In contrast, trans-fatty acids in ruminant fats have a high percentage of vaccenic acid(trans-11-C18:1 or transC18:1n-7) ${ }^{4)}$.

Epidemiological studies have shown that high dietary intake of PHVO increases low-density lipoprotein(LDL)

Abbreviations: C18:1, octadecenoic acid; C21:0-ME, heneicosanoic acid methyl ester; C21:0-TAG, triheneicosahexaen; GCFID, gas chromatography-flame ionization detector; HDL, high-density lipoprotein; LDL, low-density lipoprotein; LOD, limit of detection; LOQ, limit of quantification; ME, methyl ester; PHVO, partially hydrogenated vegetable oil; $R_{\mathrm{F}}$, response factor; $R_{\mathrm{s}}$, resolution factor; $s / n$, signal-to-noise ratio; trans9-C18:1, elaidic acid; trans-11-C18:1, vaccenic acid

\footnotetext{
*Correspondence to: Naohiro Gotoh, Department of Food Science and Technology, Tokyo University of Marine Science and Technology, 4-5-7 Konan, Minato-ku, Tokyo 108-8477, Japan

E-mail: ngotoh@kaiyodai.ac.jp

Accepted May 19, 2013 (received for review April 4, 2013)

Journal of Oleo Science ISSN 1345-8957 print / ISSN 1347-3352 online

http://www.jstage.jst.go.jp/browse/jos/ http://mc.manusriptcentral.com/jjocs
} 
and decreases high-density lipoprotein (HDL) concentration in blood ${ }^{5,6)}$, and consequently, this results in adult diseases. On the other hand, many studies prove that the intake of ruminant trans-fatty acids has no relationship to coronary heart disease, and there is still controversy concerning the difference in the effects of trans-fatty acid species on health. Furthermore, the different health effects are also pointed out in the Risk Profile Sheet published by the Ministry of Agriculture, Forestry and Fisheries in $\operatorname{Japan}^{7)}$. Therefore, it is very important to distinguish between different trans-fatty acid isomers in foods. However, no method is available to completely quantify the amount of the respective isomers for the main trans-fatty acids in foods.

The trans-fatty acids in foods are identified using a gas chromatography-flame ionization detector (GC-FID), equipped with the high-polarity column (SP-2560 column). There are several official methods such as the AOCS official method "Ce 1h-05"), AOAC (Association of Official Analytical Chemists) International official method "996.06")," and JOCS(Japan Oil Chemists' Society) official method "2.4.4.3-2013 $3^{10)}$." Although these methods can partially separate cis- and trans-C18:1 methyl ester (C18:1$\mathrm{ME}$ ) isomers, several peaks of cis- and trans-C18:1-ME isomers overlap on the chromatogram. Therefore, it is difficult to quantify a precise amount of cis- and trans-C18:1$\mathrm{ME}$ isomers in the foods. To overcome these problems, several different approaches have been used to analyze the cis- and trans-C18:1-ME isomers. To provide a definitive composition of fatty acids, a prior separation of cis- and trans-C18:1-ME isomers by silver-ion thin layer chromatography (Ag-TLC) and silver-ion high-performance liquid chromatography (Ag-HPLC) has been employed ${ }^{11,12)}$. However, this procedure is time-consuming. Recently, Goto et al. reported a simple pre-fractionation method by using silver-ion solid-phase extraction (Ag-SPE) ${ }^{13)}$. As an alternative approach without prior silver-ion fractionation, Kramer et al. separated cis- and trans-C18:1-ME isomers using different GC temperature programs. This method takes advantage of differences in the relative retention time between cis- and trans-C18:1-ME isomers, depending on the GC temperature ${ }^{14)}$. However, this tendency depends on the type of columns. Consequently, the risk of misidentification of cis- and trans-C18:1-ME isomers exists when using this method.

The AOCS official method, Ce 1h-05, is one of the conventional methods to analyze cis- and trans-C18:1-ME isomers. However, the resolution characteristics such as the resolution and response factor, elution order at various temperatures, and overlapping components of cis- and trans-C18:1-ME isomers have not yet been properly investigated. Moreover, there is not enough information on whether the contents of trans-fatty acids in foods are affected by prior separation of cis-fatty acids.
In this study, the resolution behavior and the resolution and response factors for determination of cis- and transC18:1-ME isomers were assessed by analysis of reference standards of respective cis- and trans-C18:1-ME isomers with the Ce 1h-05 method. To investigate the effect of overlapping of the peaks of cis- and trans-fatty acids, the contents of cis- and trans-C18:1-ME isomers in PHVO and milk fat were quantified by the Ce 1h-05 method and compared with those obtained by using Ag-SPE.

\section{EXPERIMENTAL}

\subsection{Chemicals and materials}

All the cis- and trans-C18:1-ME isomers used as reference materials are shown in Fig. 1 (purity: $>99 \%$ ). These C18:1-ME isomers, heneicosanoic acid methyl ester (C21:0$\mathrm{ME}$ ) and triheneicosanoin (C21:0-TAG), and other reagents were obtained from Wako Pure Chemical Industries Ltd. (Osaka, Japan). PHVO and butter were in-house products (Tsukishima Foods Industry Co. Ltd., Tokyo, Japan). Partially hydrogenated rapeseed oil having an iodine value of 71.5 was selected as a PHVO.

\subsection{Analytical conditions}

One microliter of the C18:1-ME isomer in the hexane solution was subjected to GC-FID (TRACE GC ULTRA, Thermo Fisher Scientific Inc., Waltham, MA) equipped with a cyanopropyl capillary column (SP-2560, $100 \mathrm{~m} \times 0.25 \mathrm{~mm}$ ID, $0.20 \mu \mathrm{m}$ thickness, Sigma-Aldrich Japan K. K., Tokyo, Japan). The temperatures of both the injection port and detector were maintained at $250^{\circ} \mathrm{C}$. The column temperature was maintained at $180^{\circ} \mathrm{C}$ and the split ratio was 100:1. The flow rate of the carrier gas (helium) was $1.0 \mathrm{~mL} / \mathrm{min}$.

\subsection{Resolution characteristics of cis- and trans-C18:1-ME isomers}

2.3.1 Calibration curves for C18:1-ME isomers

The calibration curves for the respective C18:1-ME isomers, limit of quantification(LOQ), and limit of detection (LOD) by the Ce $1 \mathrm{~h}-05$ method were investigated. The calibration curves were obtained by plotting the concentration ratio (C18:1-ME/C21:0-ME) on the $x$-axis and the chromatogram peak area ratio (C18:1-ME/C21:0-ME) on the $y$-axis for the respective cis- and trans-C18:1-ME isomers. The detection and quantification limit of this method were calculated by using the signal-to-noise ratio $(s / n)$. The values of LOD and LOQ were defined as $s / n=3$ and 10 , respectively ${ }^{15)}$.

2.3.2 Resolution and relative response factor

The resolution factors for the two C18:1-ME isomer peaks adjoining each other and the response factor of cisor trans-C18:1-ME isomers were examined by using a standard solution prepared by dissolving 13 types of cis- or 


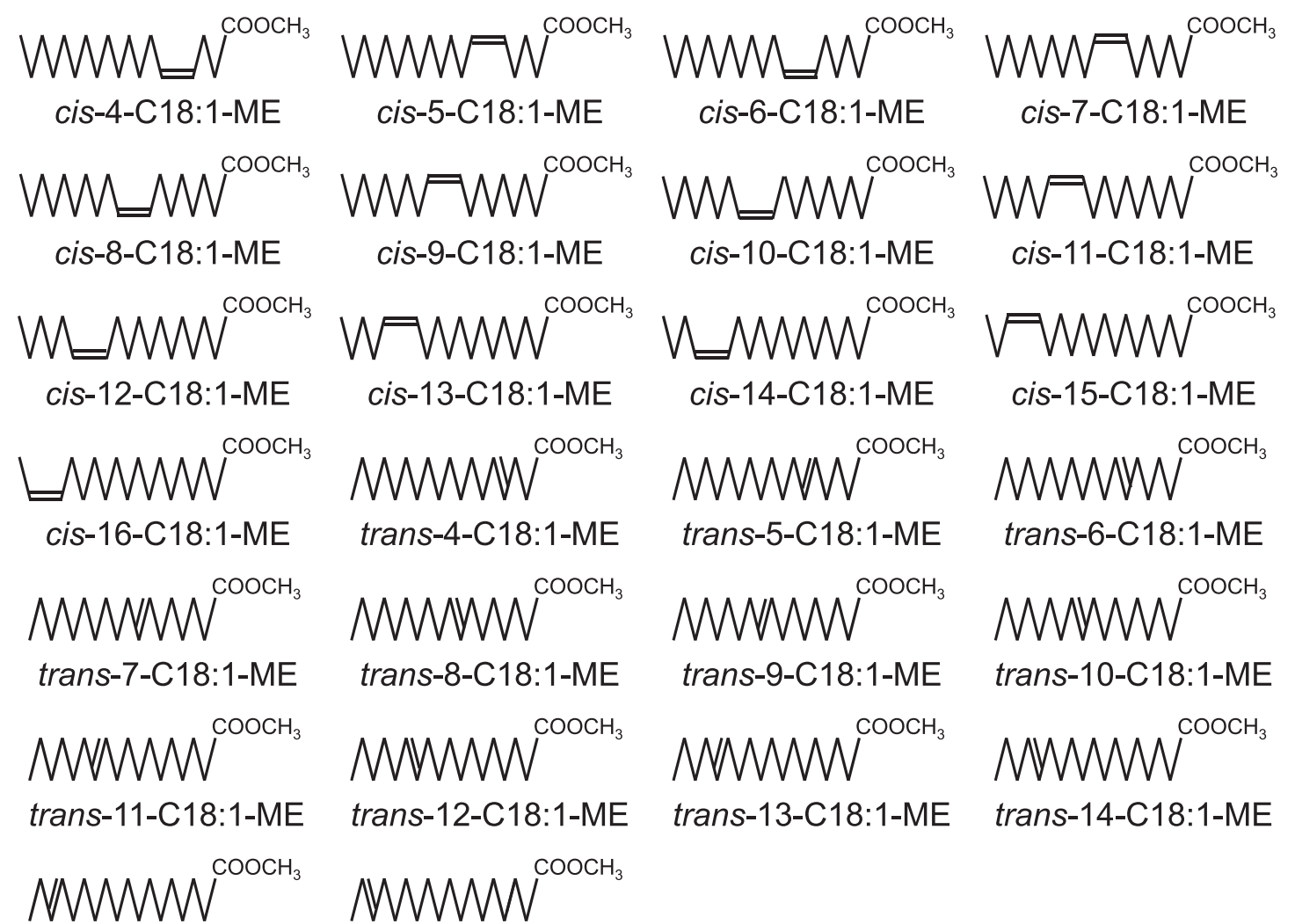

trans-15-C18:1-ME trans-16-C18:1-ME

Fig. 1 Structures of fatty acid methyl esters used in this study.

trans-C18:1-ME isomers in hexane. The standard solution also contained the C21:0-ME isomer as the internal standard.

The resolution factor $\left(R_{\mathrm{s}}\right)$ was calculated from the following equation ${ }^{16)}$ :

$$
R_{\mathrm{s}}=2\left(t_{\mathrm{R} 2}-t_{\mathrm{R} 1}\right) /\left(W_{1}+W_{2}\right),
$$

where $t_{\mathrm{R} 1}$ and $t_{\mathrm{R} 2}$ are retention times, and $W_{1}$ and $W_{2}$ are the widths of the first- and second-eluted peaks, respectively.

The relative response factor $\left(R_{\mathrm{F}}\right)$ was calculated from the following equation:

$$
R_{\mathrm{F}}=P_{\mathrm{s}} C_{\mathrm{a}} / P_{\mathrm{a}} C_{\mathrm{s}}
$$

where $P_{\mathrm{a}}$ and $P_{\mathrm{s}}$ are the peak areas and $C_{\mathrm{a}}$ and $C_{\mathrm{s}}$ are the concentrations of analyte and internal standard, respectively.

2.3.3 Elution order of cis- and trans-C18:1-ME isomers at various column temperatures

The effect of column temperature on the elution order of the respective $c i s$ - and trans-C18:1-ME isomers was investigated. The standard sample solutions were prepared by dissolving 13 types of trans-C18:1-ME isomers in hexane to obtain a final concentration of $100 \mu \mathrm{g} / \mathrm{mL}$ for the respective fatty acids. The standard solution of cis-C18:1-ME isomer was also prepared in the same way. At five different column temperatures $-170,175,180,185$, and $190^{\circ} \mathrm{C}$ - the elution orders were investigated and compared under these conditions.

\subsection{Analysis of edible fats and oils}

2.4.1 Extraction of lipids and its methyl esterification

Milk fat was extracted from butter by the Folch procedure $^{17)}$. The extracted milk fat or PHVO was methyl esterified according to the AOCS official method, Ce $1 \mathrm{~b}-89^{18)}$. $100 \mathrm{mg}$ of this sample was mixed with $1 \mathrm{~mL}$ of a $5 \mathrm{mg} / \mathrm{mL}$ solution of C21:0-TAG in chloroform in a screw-capped test tube. After the solvent was evaporated, the mixture was added to $1.5 \mathrm{~mL}$ of $0.5 \mathrm{~N}$ sodium hydroxide in methanol and heated at $100^{\circ} \mathrm{C}$ for $9 \mathrm{~min}$. The resulting solution was added to $2 \mathrm{~mL}$ of $14 \%$ solution of boron trifluoride in methanol and reheated at $100^{\circ} \mathrm{C}$ for $9 \mathrm{~min}$. After cooling the hot solution to room temperature, $1 \mathrm{~mL}$ of hexane and $5 \mathrm{~mL}$ of saturated saline solution were added to it and mixed vigorously. The upper hexane layer was moved to another screw-capped sample tube and used as the test sample solution after dehydrating with anhydrous sodium sulfate.

2.4.2 Resolution of cis- and trans-18:1-ME using silver-ion cartridge column

The resolution of cis- and trans-C18:1-ME isomers was carried out by using a silver-ion SPE cartridge ${ }^{13)}$ (Ag-ION 
SPE, Discovery Ag-ION, Sigma-Aldrich Japan K. K., Tokyo, Japan). The silver-ion cartridge column was conditioned by passing $4 \mathrm{~mL}$ of acetone followed by $8 \mathrm{~mL}$ of hexane. 0.5 $\mathrm{mg}$ of the sample was loaded into the cartridge, and $6 \mathrm{~mL}$ of dichloromethane was passed to remove the saturated fatty acid methyl ester. Then, the trans-C18:1-ME isomer was collected by using $6 \mathrm{~mL}$ of the dichloromethane and ethyl acetate mixture (90/10, v/v). Finally, the cis-C18:1$\mathrm{ME}$ isomer was collected by using $6 \mathrm{~mL}$ of the dichloromethane and ethyl acetate mixture (75/25, v/v).

2.4.3 Quantification of cis- and trans-C18:1 isomers in edible fats and oils

The cis- and trans-fatty acid isomers in PHVO and milk fat were analyzed by using the AOCS Ce 1h-05 method (Section 2.2) with or without separating the cis- and transC18:1 isomers before the analysis and by using a silver-ion cartridge column. Identification of the respective fatty acid isomers was carried out by using the retention times of the standard samples. The contents of respective fatty acids were adjusted by using the internal standard peak area and were calculated as grams per $100 \mathrm{~g}$ oil.

\section{RESULTS}

\subsection{Resolution of cis- and trans-C18:1-ME isomers by $\mathrm{Ce}$} 1h-05

The calibration curve of the trans-9-C18:1-ME isomer was plotted between 10 and $1000 \mu \mathrm{g} / \mathrm{mL}$ and was expressed as a first-order equation $\left(R^{2}>0.999\right)$. The values of LOD and LOQ were 0.40 and $0.11 \mu \mathrm{g} / \mathrm{mL}$, respectively. Other calibration curves for the C18:1-ME isomers (Fig. 1) were also constructed, all of which were expressed as a first-order equation. Their LOD and LOQ values were almost the same as those of the trans-9-C18:1-ME isomers.

The $R_{\mathrm{F}}$ values were calculated against C21:0-ME as the internal standard. For trans-C18:1-ME and cis-C18:1-ME samples, they were $1.031 \pm 0.040($ mean $\pm \mathrm{SD})$ and $0.990 \pm$ 0.032 , respectively. The $R_{\mathrm{F}}$ values for trans-isomers tend to be higher than those of cis-C18:1-ME isomers (Fig. 2). Although the values of $R_{\mathrm{F}}$ were different, the relative standard deviations for all cis- and trans-C18:1-ME isomers were $<5 \%$. The theoretical $R_{\mathrm{F}}$ values, which have been used in Ce 1h-05, is 1.0155 (dotted line in Fig. 2), which was located within the range of $R_{\mathrm{F}}$ distribution. Moreover, the average $R_{\mathrm{F}}$ for both the cis- and trans-C18:1-ME isomers obtained was $1.010 \pm 0.040$, which is approximately 1.0155 .

The elution order of trans-C18:1-ME isomers was as follows: the trans-4-C18:1-ME isomer eluted first, while the trans-16-C18:1-ME isomer eluted last(Fig. 3). However, the trans-6-C18:1-ME, trans-7-C18:1-ME, and trans-8-C18:1-ME isomers were eluted together. In addition, the peaks of trans-13-C18:1-ME and trans-14-C18:1$\mathrm{ME}$ isomers were resolved at the same time. In the case of cis-C18:1-ME isomers, it was found that the cis-7-C18:1ME eluted ahead of the cis-6-C18:1-ME isomer (data not shown). However, a good resolution could not be achieved for cis-6-C18:1-ME, cis-7-C18:1-ME, and cis-8-C18:1-ME isomers in the standard sample mixture. In addition, cis-4C18:1-ME and cis-5-C18:1-ME isomers were eluted together. The $R_{\mathrm{s}}$ values for trans- and cis-C18:1-ME isomers are shown in Tables 1 and 2, respectively. These results indicated that the pairs of trans- and cis-C18:1-ME isomers



Fig. 2 Response factors of fatty acid methyl ester samples. The dotted line (1.0155) indicates the theoretical value obtained using the AOCS official method Ce 1h-05 (mean $\pm \mathrm{SD}, n=3$ ). 


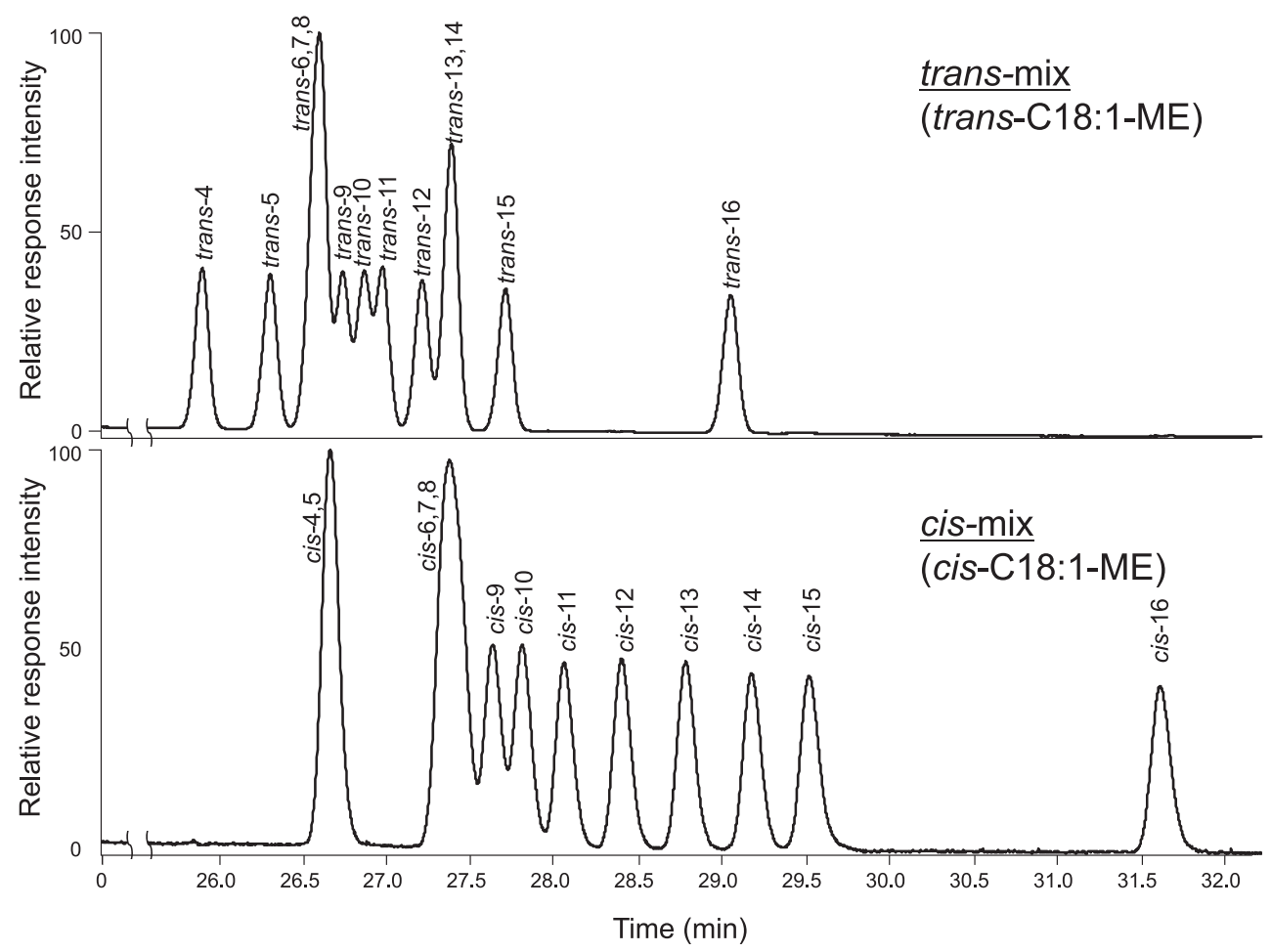

Fig. 3 GC-FID chromatograms of trans- and cis-fatty acid methyl ester samples eluted at $180^{\circ} \mathrm{C}$.

Table 1 Resolution factors of trans-C18:1-ME isomers.

\begin{tabular}{l|cccccccccccc}
\hline Double bond position & $4-5$ & $5-6$ & $6-7$ & $7-8$ & $8-9$ & $9-10$ & $10-11$ & $11-12$ & $12-13$ & $13-14$ & $14-15$ & $15-16$ \\
\hline Resolution factor & 1.91 & 1.19 & 0.14 & 0.09 & 0.65 & 0.58 & 0.54 & 1.19 & 0.71 & 0.11 & 1.61 & 6.75 \\
\hline
\end{tabular}

Table 2 Resolution factors of $c i s-\mathrm{C} 18: 1-\mathrm{ME}$ isomers.

\begin{tabular}{l|cccccccccccc}
\hline Double bond position & $4-5$ & $5-7$ & $7-6$ & $6-8$ & $8-9$ & $9-10$ & $10-11$ & $11-12$ & $12-13$ & $13-14$ & $14-15$ & $15-16$ \\
\hline Resolution factor & 0.30 & 3.21 & 0.29 & 0.30 & 0.89 & 0.87 & 1.22 & 1.72 & 1.86 & 1.89 & 1.78 & 10.41 \\
\hline
\end{tabular}

having an $R_{\mathrm{s}}$ value of $<0.30$ could not be separated on the chromatogram. The $R_{\mathrm{s}}$ value between trans-6-C18:1-ME and trans-11-C18:1-ME isomers was $<1.0$, thus, a complete resolution was not accomplished under the present analytical conditions.

\subsection{Elution order of cis- and trans-C18:1-ME isomers at various column temperatures}

The elution orders of trans- and cis-C18:1-ME isomers at at $170-190^{\circ} \mathrm{C}$ are shown in Table 3. The peaks for the following groups of isomers overlapped on the chromatogram at $180^{\circ} \mathrm{C}$ : "cis-4-C18:1-ME, cis-5-C18:1-ME, and trans-9-C18:1-ME isomers,” “cis-6-C18:1-ME, cis-7-C18:1$\mathrm{ME}$, cis-8-C18:1-ME, trans-13-C18:1-ME, and trans-14C18:1-ME isomers," and "cis-9-C18:1-ME and trans-15C18:1-ME isomers." Some of the elution orders reversed at the five different column temperatures; this indicates that co-eluting components (dotted frame in Table 3 ) are changed by GC temperature. The resolution conditions for the trans- and cis-C18:1-ME isomers were improved by lowering the column temperature; however, instead of improving the resolution, the elution times corresponding to trans- and cis-C18:1-ME isomer peaks gradually became closer.

\subsection{Contents of cis- and trans-C18:1 isomers in edible fats and oils}

The contents of the respective fatty acids in PHVO and milk fat, which are determined without the silver-ion cartridge column used for the separation of the cis-and trans-C18:1 isomers, are shown in Table 4. All the inseparable peaks of trans-9-C18:1-ME, cis-4-C18:1-ME, and cis5-C18:1-ME isomers were considered as trans-fatty acids. Similarly, all the inseparable peaks of trans-13-C18:1-ME, trans-14-C18:1-ME, cis-6-C18:1-ME, cis-7-C18:1-ME, and cis-8-C18:1-ME isomers were also considered as trans-fatty acids. In contrast, inseparable peaks of trans-15-C18:1ME and cis-9-C18:1-ME isomers were considered as cis- 
Table 3 Elution orders of respective C18:1-ME isomers at 5 kinds of column temperatures.

\begin{tabular}{|c|c|c|c|c|}
\hline $170^{\circ} \mathrm{C}$ & $175^{\circ} \mathrm{C}$ & $180^{\circ} \mathrm{C}$ & $185^{\circ} \mathrm{C}$ & $190^{\circ} \mathrm{C}$ \\
\hline$t-4$ &  & te $t-4$ & $t-4$ & $t-4$ \\
\hline$t-5$ & $t-5$ & $t-5$ & $t-5$ & $t-5$ \\
\hline $\bar{t}-\overline{6}, \overline{7}, \overline{8}$ & $t-6,7,8$ & $t-6,7,8$ & $t-6,7,8$ & $t-6,7,8$ \\
\hline$-\underline{c}-4,5$ & $c-4,5$ & $c-4, \overline{5}$ & $t-9$ & $t-9$ \\
\hline$t-9$ & $t-9$ & $-t-9$ & $c-4,5$ & $\underline{c}-\underline{4}, \underline{5}$ \\
\hline$t-10$ & $t-10$ & $t-10$ & $t-10$ & $t-10$ \\
\hline$t-11$ & $t-11$ & $t-11$ & $t-11$ & $\underline{t}-11$ \\
\hline$t-12$ & $t-12$ & $t-12$ & $t-12$ & $t-12$ \\
\hline$c-6,7,8$ & $c-6,7,8$ & $t-13,14$ & $t-13,14$ & $\bar{t}-\overline{13}, \overline{14}$ \\
\hline$t-13,14$ & $t-13,14$ & $c-6,7,8$ & $c-6,7,8$ & $c-6,7,8$ \\
\hline$c-9$ & $c-9$ & $c-9$ & $t-15$ & $t-15$ \\
\hline$-\underline{c}-10$ & $t-15$ & $\underline{t}-15$ & $c-9$ & $c-9$ \\
\hline$t-15$ & $c-10$ & $c-10$ & $c-10$ & $c-10$ \\
\hline$c-11$ & $c-11$ & $c-11$ & $c-11$ & $c-11$ \\
\hline$c-12$ & $c-12$ & $c-12$ & $c-12$ & $c-12$ \\
\hline$c-13$ & $c-13$ & $c-13$ & $c-13$ & $c-13$ \\
\hline$t-16$ & $t-16$ & $t-16$ & $t-16$ & $t-16$ \\
\hline$c-14$ & $c-14$ & $c-14$ & $c-14$ & $c-14$ \\
\hline$c-15$ & $c-15$ & $c-15$ & $c-15$ & $c-15$ \\
\hline$c-16$ & $c-16$ & $c-16$ & $c-16$ & $c-16$ \\
\hline
\end{tabular}

trans : $t$, cis : $c$

fatty acids.

Both PHVO and milk fat contained many types of cisand trans-C18:1 isomers, and the total contents of transC18:1 in PHVO and milk fat were $28.01 \mathrm{~g}$ and $3.62 \mathrm{~g}$ per $100 \mathrm{~g}$ oil, respectively, when the cis- and trans-C18:1 isomers were not separated by using a silver-ion cartridge column. When the cis- and trans-C18:1 isomers were separated before the analyses, the total contents of the transC18:1 isomer in PHVO and milk fat were $23.03 \mathrm{~g}$ and $2.78 \mathrm{~g}$ per $100 \mathrm{~g}$ oil, respectively.

\section{DISCUSSION}

\subsection{Resolution character of cis- and trans-C18:1-ME iso-} mers by Ce 1h-05

The GC-FID performance for the quantification of cisand trans- C18:1-ME isomers includes a broad range of linear response, low LOD, and LOQ. On the basis of these results, it is concluded that the sample solution adjusted to $1 \%$ for GC analysis is acceptable for analyzing $0.01 \%$ of cis- and trans-C18:1-ME isomers because the LOQ value for all the isomers studied was $0.40 \mu \mathrm{g} / \mathrm{mL}$.

The response of the FID instrument has been known to be linear, and the response behavior of the analyte depends on the number of carbon atoms and double bonds in the fatty acids. Therefore, the theoretical $R_{\mathrm{F}}$ values using Ce 1h-05 can be calculated from the structures of the respective fatty acids. However, the differences in the doublebond positions in the fatty acids and their corresponding volatility could affect their GC-FID chromatogram peak areas. In fact, the individual $R_{\mathrm{F}}$ values of cis- and transC18:1-ME isomers were slightly different, although the average $R_{\mathrm{F}}$ value for both the cis- and trans-C18:1-ME isomers was nearly the same as that obtained by employing AOCS method.

\subsection{Elution order of cis- and trans-C18:1-ME isomers at various column temperatures}

The elution order of trans- and cis-C18:1-ME isomers under AOCS Ce 1h-05 conditions (Fig. 3) are similar to the findings of a previous report ${ }^{2,19,20)}$. These orders are affected by the GC temperature. In particular, the relative retention time of cis-fatty acids is more affected than that of trans-fatty acids is. According to a previous report, the polarity of the cyanopropyl column is strongly affected by the temperature changes ${ }^{21}$. Furthermore, it is reported that compared to the trans-isomers, the cis-isomers were retained more strongly on the cyanopropyl stationary phase of the SP-2560 column when the column temperature was increased $^{22)}$.

In this study, overlapping of the peaks of some of transC18:1-ME isomers and cis-C18:1-ME isomers was observed (Table 3). This could lead to miscalculation of the total 
Table 4 Contents of cis and trans-C18:1 in PHVO and milk fat (g/100g oil).

\begin{tabular}{|c|c|c|c|c|c|c|}
\hline & \multicolumn{3}{|c|}{ PHVO (mean $\pm \mathrm{SD}, \mathrm{n}=3)$} & \multicolumn{3}{|c|}{ Milk fat (mean $\pm S D, n=3$ ) } \\
\hline & \multirow{2}{*}{$\begin{array}{c}\text { Without Ag } \\
\text { Total }\end{array}$} & \multicolumn{2}{|c|}{ With Ag } & \multirow{2}{*}{$\begin{array}{c}\text { Without Ag } \\
\text { Total }\end{array}$} & \multicolumn{2}{|c|}{ With Ag } \\
\hline & & trans & cis & & trans & cis \\
\hline$t-4-\mathrm{C} 18: 1$ & $0.12 \pm 0.00$ & $0.13 \pm 0.00$ & & $0.01 \pm 0.01$ & $0.01 \pm 0.00$ & \\
\hline$t-5-\mathrm{C} 18: 1$ & $0.23 \pm 0.00$ & $0.23 \pm 0.00$ & & $0.01 \pm 0.00$ & $0.01 \pm 0.00$ & \\
\hline$t-6,7,8-\mathrm{C} 18: 1$ & $4.09 \pm 0.01$ & $3.75 \pm 0.01$ & & $0.14 \pm 0.01$ & $0.08 \pm 0.00$ & \\
\hline $\begin{array}{r}c-4,5-\mathrm{C} 18: 1 \\
t-9-\mathrm{C} 18: 1\end{array}$ & $5.34 \pm 0.13$ & $4.42 \pm 0.01$ & $0.21 \pm 0.00$ & $0.19 \pm 0.02$ & $0.08 \pm 0.00$ & $0.18 \pm 0.03$ \\
\hline$t-10-\mathrm{C} 18: 1$ & $5.13 \pm 0.15$ & $3.92 \pm 0.00$ & & $0.15 \pm 0.01$ & $0.07 \pm 0.00$ & \\
\hline$t-11-\mathrm{C} 18: 1$ & $5.05 \pm 0.08$ & $3.43 \pm 0.00$ & & $1.81 \pm 0.05$ & $1.23 \pm 0.00$ & \\
\hline$t-12-\mathrm{C} 18: 1$ & $2.62 \pm 0.02$ & $3.14 \pm 0.01$ & & $0.20 \pm 0.01$ & $0.19 \pm 0.00$ & \\
\hline $\begin{array}{l}t-13,14-\mathrm{C} 18: 1 \\
c-6,7,8-\mathrm{C} 18: 1\end{array}$ & $4.62 \pm 0.01$ & $2.52 \pm 0.00$ & $2.44 \pm 0.06$ & $0.74 \pm 0.00$ & $0.56 \pm 0.00$ & $0.24 \pm 0.03$ \\
\hline $\begin{array}{r}c-9-\mathrm{C} 18: 1 \\
t-15-\mathrm{C} 18: 1\end{array}$ & $15.92 \pm 0.01$ & $0.68 \pm 0.00$ & $24.27 \pm 0.25$ & $21.09 \pm 0.03$ & $0.19 \pm 0.00$ & $19.23 \pm 0.13$ \\
\hline$c-10-\mathrm{C} 18: 1$ & $1.11 \pm 0.01$ & & $1.53 \pm 0.00$ & $0.00 \pm 0.00$ & & $0.00 \pm 0.00$ \\
\hline$c-11-\mathrm{C} 18: 1$ & $2.04 \pm 0.01$ & & $2.29 \pm 0.03$ & $0.48 \pm 0.01$ & & $0.43 \pm 0.02$ \\
\hline$c-12-\mathrm{C} 18: 1$ & $3.69 \pm 0.00$ & & $3.69 \pm 0.00$ & $0.18 \pm 0.01$ & & $0.18 \pm 0.00$ \\
\hline$c-13-\mathrm{C} 18: 1$ & $0.76 \pm 0.00$ & & $0.69 \pm 0.01$ & $0.07 \pm 0.01$ & & $0.07 \pm 0.00$ \\
\hline$t-16-\mathrm{C} 18: 1$ & $0.80 \pm 0.01$ & $0.80 \pm 0.00$ & & $0.36 \pm 0.00$ & $0.36 \pm 0.00$ & \\
\hline$c-14-\mathrm{C} 18: 1$ & $0.46 \pm 0.00$ & & $0.48 \pm 0.01$ & $0.00 \pm 0.01$ & & $0.02 \pm 0.01$ \\
\hline$c-15-\mathrm{C} 18: 1$ & $0.47 \pm 0.01$ & & $0.96 \pm 0.03$ & $0.12 \pm 0.01$ & & $0.08 \pm 0.00$ \\
\hline$c-16-\mathrm{C} 18: 1$ & $0.54 \pm 0.00$ & & $0.86 \pm 0.00$ & $0.08 \pm 0.01$ & & $0.07 \pm 0.00$ \\
\hline$\sum$ trans-C18:1 & 28.01 & 23.03 & & 3.62 & 2.78 & \\
\hline
\end{tabular}

trans: $t$, cis: $c$

Without Ag: Ag cartridge column was not used for the separation of cis-and trans-fatty acids before analysis

With Ag: Ag cartridge column was used for the separation of cis- and trans-fatty acids before analysis

content of trans-fatty acids in foods. In fact, the peak corresponding to elaidic acid, a trans-9-C18:1 isomer, which is the dominant trans-fatty acid species in PHVO (occupied 15.2-46.1\% of trans-fatty acid $^{2)}$ ), overlapped with several peaks on the chromatogram.

\subsection{Contents of cis- and trans-C18:1 isomers in edible fats and oils}

The total contents of trans-C18:1 in PHVO and milk fat were higher than the results of Ag-SPE analysis (28.01 g and $3.62 \mathrm{~g}$ per $100 \mathrm{~g}$ oil versus $23.03 \mathrm{~g}$ and $2.78 \mathrm{~g}$ per $100 \mathrm{~g}$ oil). It is concluded that the differences in the contents between the two methods could be ascribed to the overlapping of some of the cis-C18:1-ME isomer peaks with the peaks of the trans-C18:1-ME isomers. For example, the fatty acid content of cis-4-C18:1 and cis-5-C18:1 isomers was $0.21 \mathrm{~g}$ per $100 \mathrm{~g}$ oil, while that of cis-6-C18:1, cis-7C18:1, and cis-8-C18:1 isomers was $2.44 \mathrm{~g}$ per $100 \mathrm{~g}$ oil in PHVO (Table 4). Previous studies reported that minor cis-
C18:1 isomers ranging from cis-6 to cis-15 except cis-9 exist in PHVO and milk fat ${ }^{4,14,19)}$. The cis-C18:1 and transC18:1 isomer peaks overlapped. This could lead to miscalculation of the total trans-fatty acid content. Lowering the column temperature could improve the resolution of cisor trans-C18:1-ME isomer peaks; however, the overlapping of those peaks could lead to adverse results.

The resolution behavior and $R_{\mathrm{F}}$ values of the cis- and trans-C18:1-ME isomers were examined in this study. The results showed that the $R_{\mathrm{F}}$ values for the C21:0-ME, cisC18:1-ME, and trans-C18:1-ME isomers showed little difference among them; however, the respective values were near the theoretical value of 1.0155 , as indicated in the Ce 1h-05 method. Consequently, the Ce 1h-05 method that has been used in this study for the quantification of transfatty acid content in food was found reasonable even if all the trans-C18:1 peaks were adapted to 1.0155 for the calculation. In contrast, the resolution of cis- and trans- 
C18:1-ME isomers did not improve on changing the column temperature. The development of a method for quantifying the respective cis- and trans-C18:1-ME isomers is extremely important for revealing their effect on the lipid metabolism. Development of new methods for their resolution might be possible using higher-polarity GC columns, which is our next research target.

\section{REFERENCES}

1) Codex Alimentarius Commission, Guidelines on $\mathrm{Nu}$ trition Labelling, CAC/GL 2-1985 (1985).

2) Wolff, R. L.; Combe, N. A.; Destaillats, F. D.; Boué, C.; Precht, D.; Molkentin, J.; Entressangles, B. Follow-Up the $\Delta 4$ to $\Delta 16$ trans-18:1 Isomers Profile and Content in French Processed Foods Containing Partially Hydrogenated Vegetable Oils During the Period 19951999. Analytical and Nutritional Implications. Lipids. 35, 815-825 (2000).

3) European Food Safety Authority, Opinion of the Scientific Panel on Dietetic Products, Nutrition and Allergies on a request from the Commission related to the presence of trans fatty acids in foods and the effect on human health of the consumption of trans fatty acids (Request N-EFSA-Q-2003-022). The EFSA Journal. 81, 1-49 (2004).

4) Precht, D.; Molkentin, J.; Destaillats, F.; Wolff, R. L. Comparative Studies on Individual Isomeric 18:1 Acids in Cow, Goat, and Ewe Milk Fats by Low-Temperature High-Resolution Capillary Gas-Liquid Chromatography. Lipids. 36, 827-832(2001).

5) Zock, P. L.; Katan, M. B. Trans fatty acids, Lipoproteins, and coronary risk. Can. J. Physiol. Pharmacol. 75, 211-216 (1997).

6) Mozzaffarian, D.; Katan, M. D.; Ascherio, A.; Stampfer, M. J.; Willett, W. C. Trans fatty acids and cardiovascular disease. N. Engl. J. Med. 354, 1601-1613(2006).

7) The Ministry of Agriculture, Forestry and Fisheries of Japan. The Risk Profile Sheet about Food Safety (in Japanese). http://www.maff.go.jp/j/syouan/seisaku/ risk_analysis/priority/pdf/100728_trans_fat.pdf (accessed May 5, 2013).

8) American Oil Chemists' Society, Official Methods and Recommended Practices of the AOCS. AOCS, Official Method Ce 1h-05 (2005).

9) AOAC International, Official Methods of Analysis. AOAC International, Method 996.06 (2001).

10) Japan Oil Chemists' Society, Standard Methods for the Analysis of Fats, Oils and Related Materials. 2.4.4.3-2013 (2013)

11) Japan Oil Chemists' Society, Standard Methods for the Analysis of Fats, Oils and Related Materials (in Japanese). Recommended method 3-2013 (2013).

12) American Oil Chemists' Society, Official Methods and Recommended Practices of the AOCS. AOCS, Official Method Ce 1g-96 (2005).

13) Goto, H.; Shionoya, N.; Sugie M.; Tominaga, M.; Shimelis, O.; Taniguchi, M.; Igarashi, T.; Hirata, Y. Novel Prefractionation Method of Trans Fatty Acids by Gas Chromatography with Silver-Ion Cartridge Column. J. Oleo Sci. 61, 49-56 (2012).

14) Kramer, J. G.; Hernandez, M.; Cruz-Hernandez, C.; Kraft, J.; Dugan, M. E. Combining Results of Two GC Separations Partly Achieves Determination of All cis and trans 16:1, 18:1, 18:2, and 18:3 Expect CLA Isomers of Milk Fat as Demonstrated Using Ag-Ion SPE Fractionation. Lipids. 43, 259-273 (2008).

15) Hernandez, M. J.; Garcia-Moreno, M. V.; Duran, E.; Guillen, D.; Barroso, C. G. Validation of two analytical methods for the detection of ochratoxin A by reversed-phased high-performance liquid chromatography coupled to fluorescence detection in musts and sweet wines from Andalusia. Anal. Chim. Acta 566, 117-121 (2006).

16) Reich, E.; Schibli, A. High-Performance Thin-Layer Chromatography for the Analysis of Medical Plants. New York: Thieme. p 31 (2006).

17) Folch, J.; Lees, M.; Sloane-Stanley, G. H. A simple method for the isolation and purification of total lipids from animal tissues. J. Biol. Chem. 226, 497-509 (1957).

18) American Oil Chemists' Society, Official Methods and Recommended Practices of the AOCS. AOCS, Official Method Ce 1b-89 (1997).

19) Wolff, R. L.; Bayard, C. C. Improvement in the Resolution of Individual trans-18:1 Isomers by Capillary GasLiquid Chromatography: Use of a 100-m CP-Sil 88 Column. J. Am. Oil. Chem. Soc. 72, 1197-1201 (1995).

20) Ratnayake, W. M. N.; Plouffe, L. J.; Pasquier, E.; Gagnon, C. Temperature-Sensitive Resolution of cis- and trans-Fatty Acid Isomers of Partially Hydrogenated Vegetable Oils on SP-2560 and CP-Sil 88 Columns. J. AOAC Int. 85, 1112-1118(2002).

21) Thompson, R. H. Direct Measurement of Total transand cis-Octadecenoic Fatty Acids Based on a Gas-Liquid Chromatographic Class Separation of trans-18:1 and cis-18:1 Fatty Acid Methyl Esters. J. Chromatogr. Sci. 35, 536-544 (1997).

22) Mjøs, S. A. Properties of trans Isomers of Eicosapentaenoic Acid and Docosahexaenoic Acid Methyl Esters on Cyanopropyl Stationary Phases. J. Chromatogr. A. 1100, 185-192(2005). 